\title{
Modeling the Adverse Impact of Rainstorms on a Regional Transport Network
}

\author{
Saini Yang ${ }^{1,2} \cdot$ Guofan $\mathrm{Yin}^{1,2} \cdot \mathrm{Xianwu} \mathrm{Shi}^{3} \cdot \mathrm{Hao} \mathrm{Liu}^{4} \cdot$ Ying Zou $^{5}$
}

Published online: 21 March 2016

(c) The Author(s) 2016. This article is published with open access at Springerlink.com

\begin{abstract}
Cities are centers of socioeconomic activities, and transport networks carry cargoes and passengers from one city to another. However, transport networks are influenced by meteorological hazards, such as rainstorms, hurricanes, and fog. Adverse weather impacts can easily spread over a network. Existing models evaluating such impacts usually neglect the transdisciplinary nature of approaches for dealing with this problem. In this article, a mesoscopic mathematical model is proposed to quantitatively assess the adverse impact of rainstorms on a regional transport network in northern China by measuring the reduction in traffic volume. The model considers four factors: direct and secondary impacts of rainstorms, interdependency between network components, and recovery abilities of cities. We selected the Beijing-Tianjin-Hebei region as the case study area to verify our model. Socioeconomic, precipitation, and traffic volume data in this area were used for model calibration and validation. The case study highlights the potential of the proposed
\end{abstract}

Saini Yang

yangsaini@bnu.edu.cn

$1 \quad$ State Key Laboratory of Earth Surface Processes and Resource Ecology, Beijing Normal University, Beijing 100875, China

2 Academy of Disaster Reduction and Emergency Management, Beijing Normal University, Beijing 100875, China

3 National Marine Hazard Mitigation Service, Beijing 100194, China

4 Beijing Transportation Information Center, Beijing 100161, China

5 Beijing Municipal Commission of Transport, Beijing 100073, China model for rapid disaster loss assessment and risk reduction planning.

Keywords China - Disaster loss assessment . Mathematical modeling - Rainstorm hazards . Recovery $\cdot$ Transport networks

\section{Introduction}

Transport networks are a major component of critical infrastructures and essential for the efficient and organized operation of our society and economy. By effectively moving people and cargo from one place to another, traffic flows between cities play a similar role in a socioeconomic system as the circulation system does in the human body. But transport networks are vulnerable to meteorological hazards, such as rainstorms, hurricanes, and fog. Because of the interdependency between network components, any adverse influence can spread from one city to another, even through the whole network (Johansson and Hassel 2010), causing economic losses in local, regional, or even global systems. In the context of global change, the occurrence of extreme weather events might substantially increase (Rahmstorf and Coumou 2011), resulting in higher weather-related risks.

The heavy snowfall in Europe from 2010 to 2011, for example, resulted in massive congestions and disruptions on a very large scale. The UK experienced the coldest, record-breaking December in 100 years (WMO 2011). Many highways were blocked, countless flights were canceled, and regional traffic was almost paralyzed.

In the face of such hazardous weather events, an impact assessment model is urgently needed by administrative agencies for various purposes, including traffic management, transportation planning, rapid disaster loss assessment, and 
disaster risk reduction. Given the interdisciplinary nature of the problem, such a model requires knowledge from multiple fields, such as disaster risk, transportation engineering, and network systems.

Research in transportation engineering has mainly focused on the relationship between adverse weather intensity and traffic flow parameters. Smith et al. (2004) analyzed the impact of different precipitation intensities on various freeway parameters from traffic and weather data for the Hampton Roads region of Virginia in the United States. Dehman (2012) investigated the pre-breakdown flow and queue discharge flow of four bottlenecks in Milwaukee, Wisconsin in the United States, under different weather conditions and proposed the use of weather reduction factors in their models. Weng et al. (2013) compared traffic flow under normal conditions and under snow conditions and quantitatively described the relationship between traffic flow parameters and adverse weather. Chung (2012) analyzed the effect of precipitation on traffic congestion and proposed a function to describe the average nonrecurrent traffic congestion per unit distance. Saberi and Bertini (2010) also analyzed the relationship between traffic and weather, and found that different rainfall conditions had distinct impacts on traffic speed and flow during congested and uncongested periods. Camacho et al. (2010) assessed effects on traffic for four different weather types with nonlinear regression methods. Researchers usually concentrate on analyzing quantitatively the relationship between adverse weather and traffic parameters. They seldom consider the intrinsic properties of the network system, such as its interdependencies and recovery. The complete cycle of an adverse impact, that is, the process of emergence, development, and dissipation, also is generally not modeled.

In the field of network systems, the focus is mainly on the common characteristics of these systems. Many studies have defined parameters to characterize the metrics of networks. Rinaldi et al. (2001) highlighted the trend toward greater interdependencies between infrastructure components and proposed a taxonomy to facilitate research. Henry and Ramirez-Marquez (2012) described the metrics of network and system resilience and proposed generic metrics and formulas for quantifying system resilience. Buzna et al. (2006) proposed a synthetic model to simulate the dynamic spreading of failures in networked systems and analyzed the system properties under different circumstances. Attoh-Okine et al. (2009) proposed a resilience index for urban infrastructures using belief functions. Amin (2000) illustrated enhanced interdependency between components of infrastructure networks and outlined the common characteristics that make them difficult to control and operate reliably and efficiently. Due to simplifications in these models, characteristics of a specific system are often ignored and real-world case studies are limited.

Researchers in the disaster risk field have made progress in modeling the impacts of meteorological hazards. Su et al. (2016) established an integrated simulation method of rainfall-affected traffic in a city, in which the waterlogging effect was considered. Chang et al. (2014) developed a practical approach to characterizing community infrastructure vulnerability and resilience to disasters. Godschalk (2003) proposed a comprehensive strategy for urban hazard mitigation, aimed at creating resilient cities that are capable of withstanding both natural and terroristic hazards. Santos et al. (2014) discussed the need for a paradigm shift in disaster risk analysis to emphasize the role of the workforce in managing the recovery of interdependent infrastructures and economic systems. Chang et al. (2007) used a conceptual model to investigate infrastructure failures caused by power outages, resulting from interdependencies. So far, there are few quantitative methods or models available for the impact assessment of meteorological hazards on regional transport networks. To the best of our knowledge, there is no existing model that explicitly and simultaneously takes into account direct and secondary impacts of meteorological hazards, interdependency between network components, and the recovery ability of a system.

In this study, we combined the knowledge from the fields of disaster risks, transportation engineering, and network systems in order to benefit from their individual advantages and developed a mesoscopic model to assess the adverse impact of rainstorms through quantitatively estimating traffic volume in a regional transport network. The proposed impact assessment model takes into account the direct and secondary (waterlogging) impact of rainstorms on transport infrastructures, as well as the indirect effect caused by network connectivity.

After outlining the case study area and data sources, the article presents the proposed methodology and the key factors considered in the model, and illustrates the case study and parameter analyses. Finally, we put forward directions for further study.

\section{Study Area and Data Sources}

The Beijing-Tianjin-Hebei region is located on the North China Plain and encompasses 13 cities: Beijing, Tianjin, Baoding, Langfang, Cangzhou, Qinhuangdao, Tangshan, Chengde, Zhangjiakou, Hengshui, Xingtai, Handan, and Shijiazhuang (Fig. 1). The region covers a total area of $216,000 \mathrm{~km}^{2}$ with a population of about 110 million. Famous for its automobile, electronics, and machinery industries, the region is vital to China's booming economic 


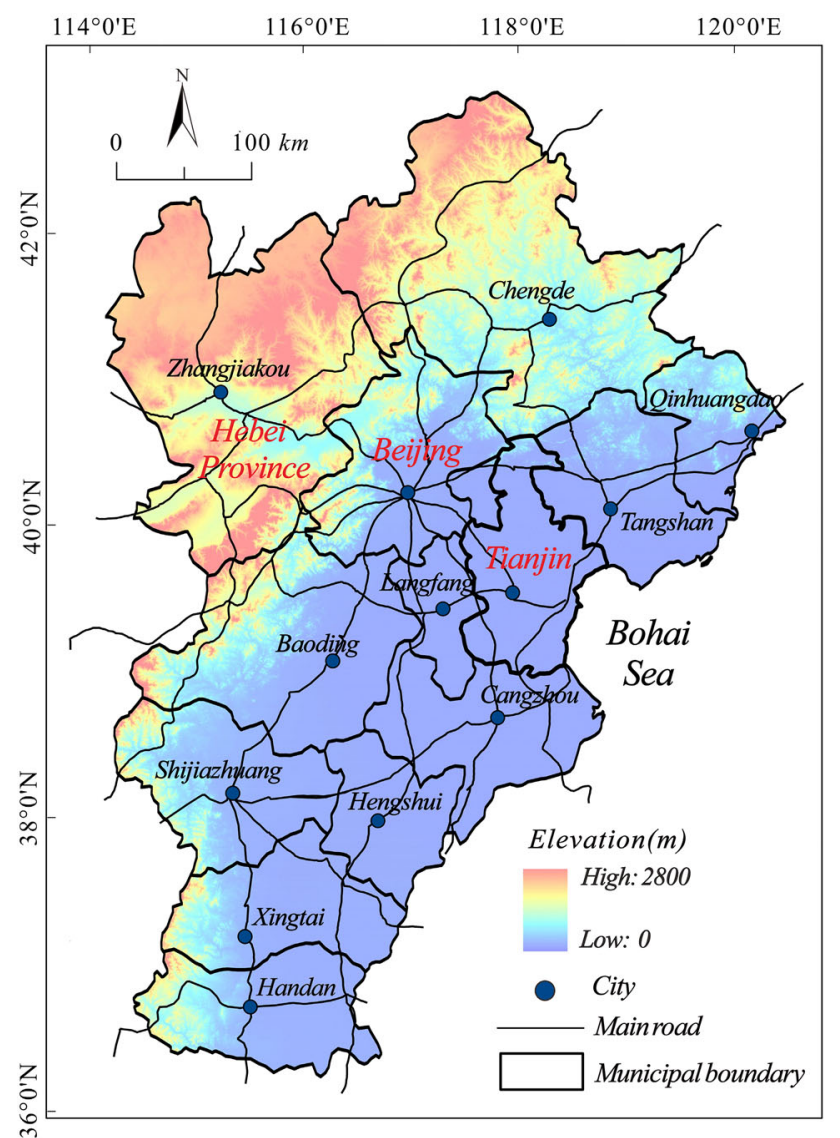

Fig. 1 The Beijing-Tianjin-Hebei study area in northern China

development, and its gross domestic product (GDP) reached USD 1 trillion in 2014 (National Bureau of Statistics of China n.d.). The accompanying urbanization process has been remarkable over the past 20 years and has resulted in rapid growth of critical infrastructures and traffic volumes. In the context of global climate change, extreme events in the Beijing-Tianjin-Hebei region have massive impacts on its infrastructure and economy. The region has become a hotspot for researchers interested in climate change adaptation and disaster risk reduction, especially since the 21 July 2012 rainstorm.

Rainstorms are a major type of natural disaster and happen frequently around the world. Losses caused by rainstorms can be tremendous in a developed socioeconomic system. On 21 July 2012, strong rainstorms hit Beijing and the surrounding areas (Guo et al. 2015). Beijing experienced a severe storm that resulted in inundation and traffic congestion. Fangshan, one of the administrative districts of Beijing, suffered the strongest rainfall from 10 a.m., 21 July to 6 a.m., 22 July, with an estimated precipitation of $460 \mathrm{~mm}$ - the heaviest in 61 years (Jiang et al. 2014). This rainstorm caused severe inundation along 63 roads, serious collapse of 31 roads, and temporary closure of 12 subway entrances. More than 400 airline flights were canceled or delayed and about 80,000 travelers were stranded at the regional airports. By 6 August 2012, 79 people had died, the daily lives of about 1.6 million people had been severely affected, and more than 50,000 people had to be transferred to safer places. This meteorological hazard contributed to a crucial traffic breakdown, both in urban and suburban areas of Beijing, with direct and indirect economic losses totaling about USD 1.88 billion (China News n.d.). We selected this extreme event as the case to evaluate our proposed model.

To analyze the adverse impact of the 21 July 2012 storm on the transport network of the region, we acquired the daily precipitation data from April 2012 to October 2012 from the weather stations located in the 13 cities in the case study area. Traffic flow data on highways were acquired from toll stations at highway entrances in the Beijing Metropolitan area for the same time period. Due to data access restrictions, we could not obtain the traffic data of all 13 cities, but only those surrounding the Beijing Metropolitan area. Because Beijing experienced the heaviest precipitation during this rainstorm and the traffic volume generated and attracted by Beijing is significantly larger than that in other cities, the accuracy of the simulation results for Beijing is of great importance to the overall accuracy of the model. The average travel times between any two cities in the case study area under normal conditions were obtained from the Baidu Map (Baidu Map n.d.), which provides reliable regional transport network information and road management parameters. The socioeconomic data for each city, such as highway passenger traffic (HPT), highway freight traffic, and GDP were obtained from the website of the National Bureau of Statistics of China (n.d.). Highway passenger traffic is defined by the number of people exported from a city through highways during a certain period. We used the value of HPT to represent intercity traffic volume under normal conditions.

\section{Methodology}

A regional transport network consists of cities and the roads that connect them. Cities can be regarded as network nodes, while roads can be regarded as their links. Given that traffic flow travels between cities on roads, we use the term "traffic volume" to represent the number of vehicles exported from one city to another during a certain period. We developed a model to quantitatively assess the impact of rainstorms on a regional transport network. We evaluated the adverse impact using the change in traffic volume of every city in a region, but not the change in transport volume of every road. 


\subsection{Factors Considered in the Modeling Process}

To satisfy conditions of accuracy and computational efficiency, a mesoscopic model is proposed, in which four factors are considered: the direct impact of rainstorms; the secondary impact (waterlogging) caused by rainstorms; the interdependency between network components; and the recovery abilities of cities. Direct impact of rainstorms refers to the traffic volume loss caused by reduced visibility and surface friction in each city. Secondary impact of heavy precipitation refers to the influence of accumulated water on road surfaces (AWORS) and the traffic breakdowns caused by severe waterlogging on traffic volume. The interdependency between network components makes it possible for traffic volume reduction to spread between cities. The recovery ability represents the capacity of a city to recover and return to a normal state of traffic volume.

\subsection{Mechanisms of the Proposed Model}

A complete cycle of adverse impact on a regional transport network includes hazard emergence, development, and dissipation stages. When traffic flows between cities, the origin city exports its traffic volume, and the destination city gains traffic volume, which can be exported to other cities later on. The traffic volume of the destination city can be regarded as increasing, and the volume of the origin city can be regarded as decreasing. When rainstorms occur, the traffic volume of each city is immediately affected due to decreased visibility and road surface friction. As the rainstorm continues, the AWORS correlated with the intensity of the precipitation, and city drainage efficiency, triggers secondary impact, such as breakdown of traffic flow. As traffic flow progresses from one city to another over the connecting roads, the adverse impact of a rainstorm spreads through the decrease in traffic volume. The recovery ability of a transport network determines its potential to recover from the adverse impact and resume increased volume. The framework of the proposed model is illustrated in Fig. 2. The sections below elaborate on how these mechanisms were abstracted as mathematical equations and Sect. 4 discusses how these equations were calibrated.

\subsubsection{System Status}

In this study, the change of a system's status is directly reflected in the change of traffic volume. The volume of city $i$ at time $t+1$ consists of the imported traffic flow from adjacent cities and the recovered volume from the adverse impact at time $t$. It is also correlated with the direct and secondary impacts of the precipitation. As precipitation is ongoing, when the depth of the AWORS exceeds a given threshold determined by tire size, vehicle strands and traffic volume reduce to zero. This threshold is referred as the "breakdown threshold" $(B)$.

$$
\begin{aligned}
& x_{i}(t+1) \\
& \quad= \begin{cases}0, & W_{i}(t) \geq B \\
{\left[G_{i}(t)+R_{i}(t)\right] *\left(1-D R_{i}\left(P_{i}(t)\right)\right),} & W_{i}(t) \geq B .\end{cases}
\end{aligned}
$$

In Eq. $1, x_{i}(t)$ denotes the traffic volume of city $i$ at time $t$. $B$ denotes the threshold, while $W_{i}(t)$ denotes the depth of the AWORS of city $i$ at time $t . G_{i}(t)$ denotes the exported traffic volume of adjacent cities to city $i$ at time $t$, and $R_{i}(t)$ denotes the recovered traffic volume of city $i$ from the adverse impact. $P_{i}(t)$ denotes the intensity of precipitation in city $i$ at time $t$, and $D R_{i}\left(P_{i}(t)\right)$ denotes the loss ratio of traffic volume with given precipitation intensity, which can be deemed as the direct adverse impact of precipitation on city $i$.

\subsubsection{Direct Impact of Precipitation on Transport Networks}

When rainstorms affect a transport network, they directly and adversely affect its normal functioning because of the reduced visibility and road surface friction. Drivers will reduce speed and increase headways in unfavorable driving conditions, and thus reduce the traffic volume of a city. Studies have illustrated the relationship between precipitation intensity and traffic speed (Yang et al. 2012; Su et al. 2016). In this study, $D R_{i}\left(P_{i}(t)\right)$ represents the direct impact suffered by city $i$, which is the ratio of the lost traffic volume at time $t$ to the average traffic volume under normal conditions. The calibration of $D R_{i}(\cdot)$ is covered in Sect. 4.1.

\subsubsection{Secondary Impact of Precipitation on Transport Networks}

The secondary impact of precipitation is caused by waterlogging. We use the depth of AWORS to represent the overall severity of waterlogging in a city. The AWORS depth at time $t+1$ consists of the amount of AWORS and precipitation at time $t$ with respect to the drainage efficiency of the city. We assume that in a city, when the AWORS is shallow, the drainage system will rapidly dissipate the accumulated water. As the amount of AWORS increases, the drainage process requires more time. When the depth of the AWORS exceeds the threshold $(B)$, the traffic volume in the city reduces to zero.

$W_{i}(t+1)=d_{i}\left(W_{i}(t)+P_{i}(t)\right)$.

In Eq. $2, d_{i}(\cdot)$ denotes the drainage efficiency of city $i$, while $(\cdot)$ is the depth of the AWORS at time $t$. 
Fig. 2 Framework of the model proposed to assess the adverse impact of rainstorms on a regional transport network

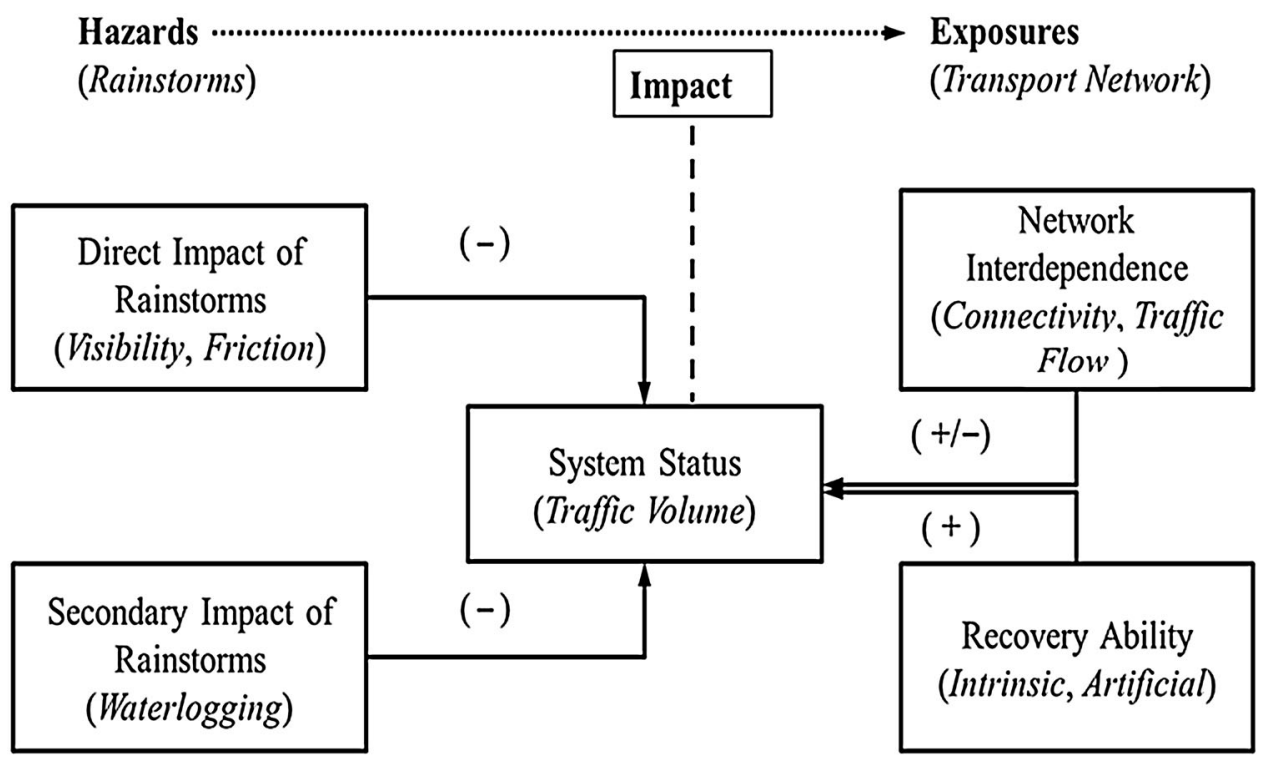

\subsubsection{Interdependency Between Cities}

Traffic flow in a network spreads from one city node to another through the "links" connecting cities. The traffic volume between each origin-destination (OD) pair can be estimated quantitatively using a gravity model (Hoel et al. 2007). Our model is determined by the attractiveness of each destination node, the impedance between origin and destination, the overall exported traffic volume of the origin node, and an intrinsic adjustment matrix. Attractiveness, often represented by gross domestic product (GDP) or annual traffic volume, denotes the ability of a destination node to attract traffic flow from an origin node. Impedance denotes the degree of difficulty in traffic flow between origin and destination, often represented by travel time or travel distance.

$G_{i}(t)=\sum_{j=1}^{N} Q_{j i}(t)$,

$Q_{j i}(t)=x_{j}(t) * \frac{\frac{A_{i} * K_{j i}}{\left(t_{j i}\right)^{2}}}{\sum_{1=1}^{N} \frac{A_{i} * K_{j i}}{\left(t_{j i}\right)^{2}}}$.

In Eq. 3.1, $N$ denotes the number of cities, $Q_{j i}(t)$ denotes the traffic volume to city $i$ from city $j$ at time $t$, determined by the gravity model expressed in Eq. 3.2. In Eq. 3.2, $A_{i}$ denotes the attractiveness of city $i, t_{j i}$ denotes the impedance between city $j$ and city $i$, and $K_{j i}$ is an intrinsic adjustment matrix in the gravity model, and usually represents the socioeconomic characteristics of city $i$ and city $j$.

\subsubsection{Recovery Ability}

The recovery ability of a system is composed of intrinsic recovery ability and artificial recovery ability. Intrinsic recovery ability refers to the ability of a system to spontaneously absorb external disturbances and return to its normal state. This type of recovery ability can be considered constant. Artificial recovery ability is closely related to the level of emergency management and planning that determines the input of resources for emergency response. When an external disturbance is large, artificial recovery ability (input of resources for emergency response) normally will increase until it reaches its limit. In a transport system, when a minor accident happens and forms a queue of delayed vehicles, for example, the queue usually dissipates automatically without any external aid. When the congestion is severe, police will show up to organize the traffic. The worse the congestion becomes, the more external aids (manpower and facilities) the system will receive.

Inspired by Hooke's Law, we assumed that the recovery ability of a city is positively correlated with the degree of the adverse impact. Recovery ability rises, as the intensity of the adverse impact increases. The degree of adverse impact is represented by the loss ratio of traffic volume, which is 1 minus the ratio of the current traffic volume to the average traffic volume under normal conditions. We assumed that the recovered volume does not exceed the difference between the current volume and the average volume without adverse impact.

$R_{i}(t)=r * P O_{i}(t) * R E_{i}\left(1-\frac{x_{i}(t)}{M A X_{i}}\right)$, 
$P O_{i}(t)=M A X_{i}-G_{i}(t)$.

In Eqs. 4.1 and 4.2, $R_{i}(t)$ is the recovered traffic volume, $r$ is an adjustment coefficient and $M A X_{i}$ denotes the average traffic volume of city $i$ without the adverse impact of a rainstorm. $R E_{i}\left(1-\frac{x_{i}(t)}{M A X_{i}}\right)$ denotes the recovery coefficient of city $i$ at time $t$, where $1-\frac{x_{i}(t)}{M A X_{i}}$ is the loss ratio of traffic volume at time $t$ of city $i$, which indicates the intensity of an adverse impact. $P O_{i}(t)$ is the difference between the incoming traffic of city $i$ at time $t$ and the average traffic volume for city $i$, which is used to restrict the recovered volume.

\section{Case Study}

In this section, we use the precipitation and traffic flow data we obtained to calibrate and evaluate our model, and analyze and discuss our simulation results.

\subsection{Selection and Calibration of Functions}

For the proposed model, basic functions of the relationships between loss ratio of traffic volume and precipitation; depth of AWORS and drainage efficiency; and loss ratio of traffic volume and recovery coefficient were first determined based on Chung et al. (2006), the Bernoulli equation of fluid dynamics, and the principle of Hooke's Law, respectively. The precipitation and traffic data from 1 April to 5 July 2012 were then used to calibrate these basic functions, and the data from 6 July to 5 August 2012 were used for model validation.

The direct adverse impact of heavy precipitation varies with cities, though the direct impact is usually within some range (Yang et al. 2012). The relationship between loss ratio of traffic volume and precipitation was derived based on the work of Chung et al. (2006) and the model parameter was calibrated using the daily precipitation data and traffic flow data for Beijing highways from 1 April 2012 to 5 July 2012, as shown in Fig. 3 The loss ratios when $P_{i}(t)$ exceeds $120 \mathrm{~mm}$ were assumed to be the same because (1) existing studies rarely analyze the influence of daily precipitation with such high intensities as those of the summer 2012 event; and (2) in our datasets, daily records exceeding $120 \mathrm{~mm}$ accounted for less than $1 \%$ of the total record numbers.

The secondary impact of precipitation is determined by the depth of the AWORS and the threshold $(B)$. Because our model is a mesoscopic model and aimed at rapid assessment of the adverse impact on a regional transport network, each city is treated as a node. We did not use a

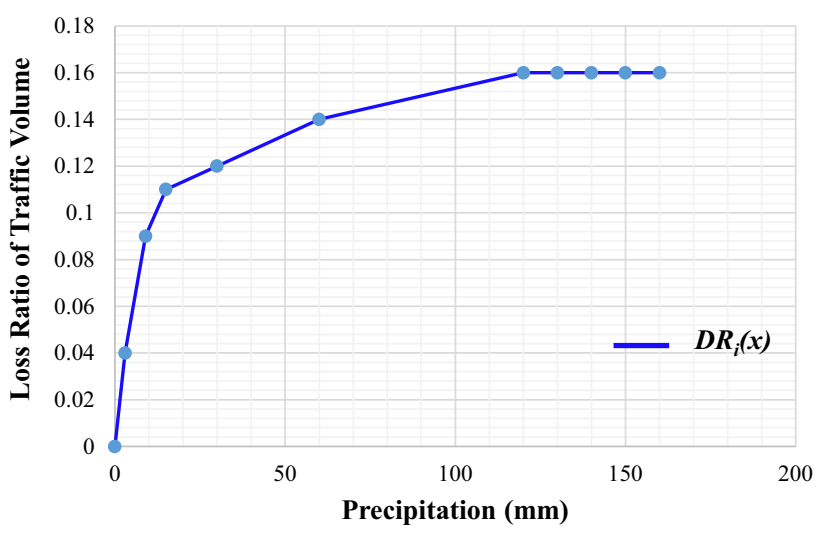

Fig. 3 Direct influence of precipitation $\left(D R_{i}(x)\right)$ on traffic volume reduction

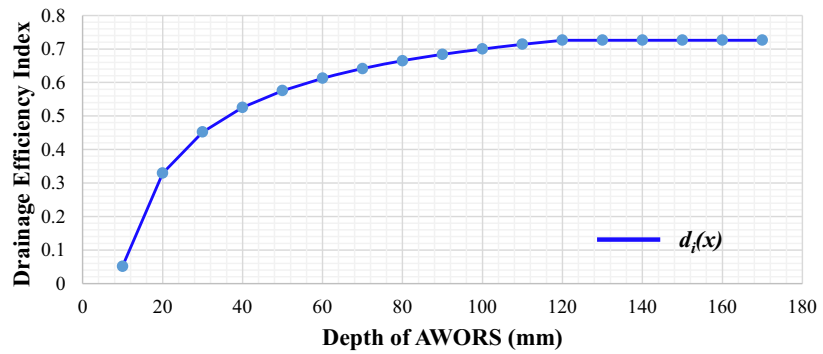

Fig. 4 Relationship between the depth of accumulated water on road surfaces (AWORS) and drainage efficiency $\left(d_{i}(x)\right)$

storm water management model (SWMM) to simulate the generation and transport of runoff from urban areas, which requires high resolution digital elevation model (DEM) data and hydrological data, and long computational time. Instead we used drainage efficiency to reflect the overall waterlogging condition of a city.

Drainage efficiency is represented by the ratio of remaining water depth to the maximum AWORS depth at a certain time. Based on the Bernoulli equation (Munson et al. 2006), which can be considered as a statement of the conservation of energy principle appropriate for flowing fluids, the speed of flow is proportional to the square root of water pressure. The relationship between drainage efficiency and AWORS is nonlinear. We chose a function of parabola-like form to represent drainage efficiency (Fig. 4), in which when the depth of AWORS exceeded $120 \mathrm{~mm}$, drainage efficiency was assumed to be the same because the drainage system was always overwhelmed. The value of $B$ depends on tire diameters, with a typical range between 540 and $840 \mathrm{~mm}$. We assumed the ratio of $B$ to the tire diameter was $1 / 3$. Consequently, $B$ in Eq. 1 was set to $230 \mathrm{~mm}$, which is $1 / 3$ of the mean tire diameter.

Because the recovery ability of a system is composed of intrinsic recovery ability, which is relatively stable, and 


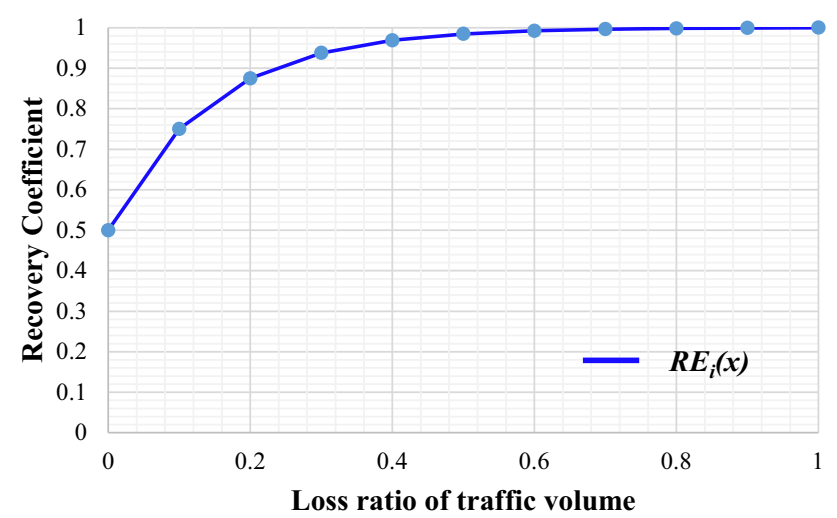

Fig. 5 Relationship between the traffic volume loss ratio and the recovery coefficient $\left(R E_{i}(x)\right)$

artificial recovery ability, which increases with the intensity of adverse impact, the recovery coefficient takes a similar form. As shown in Fig. 5, the recovery coefficient takes an initial value (intrinsic recovery) when there is no disturbance, but the value of the recovery coefficient increases as the loss ratio of traffic volume $\left(1-\frac{x_{i}(t)}{M A X_{i}}\right)$ increases (artificial recovery).

The parameters are also calibrated using the traffic flow data of Beijing highways from 1 April to 5 July 2012 (Fig. 5). To calculate the recovered volume, the adjustment parameter $r$ in Eq. 4.1 was set to 0.4 based on expert knowledge and preliminary studies.

In our case study, due to data limitations, we assumed that $D R_{i}(x), d_{i}(x)$, and $R E_{i}(x)$ were the same for all cities.

A discrete simulation program was developed using Java language. We initially assumed that there was no accumulated water in all cities, that is, $W_{i}(0)=0$. The adjustment matrix for the gravity model, $K$, was represented by a unit matrix, where elements on the diagonal were all set to 0 . Consequently, the flow of traffic originating from and targeting the same city was excluded. The attractiveness of destination cities, $A_{i}$, was represented by their annual highway passenger traffic (HPT) and the average traffic volume for each city, $M A X_{i}$ was also approximately represented by their average daily HPT of the second quarter of 2012, when there were few meteorological hazards influencing traffic. The travel impedance between each origin-destination pair is represented by the travel time obtained from the Baidu Map (Baidu Map n.d.). We set the initial traffic volume for each city $\left(x_{i}(0)\right)$ according to the results of the gravity model (Eq. 3.2) under normal conditions. The gravity model algorithm is called to determine the matrix of the traffic volume traveling from the origin node to each of the other nodes. The algorithm is described in Hoel et al. (2007). During the calculation process, the HPT of each city was set to its original volume. Through iterations of the gravity model with preset $A_{i}, t_{i j}$, and $K$, the volume for each city converged, and this value was used as the initial volume. Table 1 presents an overview for key variables in the proposed model.

\section{Results}

We implemented the proposed model in Java for each of the 13 cities in the Beijing-Tianjin-Hebei region, yielding a negligible execution time for this case study, which demonstrates high computational efficiency. To compare all the cities' aggregated difference between traffic data collected by the toll stations and the simulation results, we normalized each time series using Eq. 5, where NTS denotes the normalized time series and $T S$ denotes the raw time series. Here, $i=\{1,2\}$ denotes the type of traffic data, that is, the data collected by the toll stations or the simulation results, and $\operatorname{MAX}(x)$ is the maximum value in each time series. The relative error for these calculations is given by Eq. 6, in which $R(t)$ denotes the observed traffic volume at time $t$ and normalized $S(t)$ denotes the simulated traffic volume at time $t$. The normalized results and the relative error are given in Fig. 6.

$N T S_{i}(t)=\frac{T S_{i}(t)}{\operatorname{MAX}\left(T S_{i}(t)\right)}$,

$E(t)=\frac{R(t)-S(t)}{R(t)}$.

As shown in Fig. 6, the results generated by our proposed model differ slightly from the observed traffic volume values, and the overall trends of these two time series match. The maximum relative error is $21.7 \%$ on 9 July, and in $13 \%$ of the 31 days between 6 July and 5 August relative error exceeded $10 \%$. The average error was $5.5 \%$, but in $55 \%$ of the days relative error was lower than $5 \%$, suggesting reasonable accuracy of the proposed model.

\section{Discussion}

In this model, $r$ (adjustment parameter) and $B$ (breakdown threshold) are empirical parameters, which have not been extensively researched. We conducted parametric analyses for these variables, using nine settings (Fig. 7). The range of $r$ is about $\pm 50 \%$, while that for $B$ is about $\pm 22 \%$. Because $r$ is the adjustment parameter for quantifying the recovery ability of a city, we tested a relatively large range of values. The value of $B$ was assumed to be $1 / 3$ of tire diameters. Since most tire diameters range from 540 to $840 \mathrm{~mm}$, the range of $B$ is determined in the sensitivity analyses. 
Table 1 Model variables

\begin{tabular}{|c|c|c|}
\hline Variable name & Interpretation & Value \\
\hline$x_{i}(t)$ & Traffic volume of each city & Time-dependent \\
\hline$W_{i}(t)$ & AWORS of each city & Time-dependent \\
\hline$B$ & Breakdown threshold & $230 \mathrm{~mm}$ \\
\hline$G_{i}(t)$ & $\begin{array}{l}\text { Input volume to one city from its nearby } \\
\text { cities }\end{array}$ & Time-dependent \\
\hline$R_{i}(t)$ & Recovery ability of each city & Time-dependent \\
\hline$P_{i}(t)$ & Precipitation intensity of each city & Time-dependent \\
\hline$D R_{i}(\cdot)$ & Direct adverse impacts of precipitation & Figure 3 \\
\hline$d_{i}(\cdot)$ & Drainage efficiency of each city & Figure 4 \\
\hline$Q_{j i}(t)$ & Traffic flow transfer from one city to another & Time-dependent \\
\hline$N$ & Number of cities & 13 \\
\hline$A_{i}$ & Attractiveness of each city & Represented by the 2012 annual HPT of each city \\
\hline$t_{i j}$ & $\begin{array}{l}\text { Transportation impedance between one city } \\
\text { and another }\end{array}$ & Represented by the transportation time \\
\hline$K$ & Adjustment matrix in the gravity model & The matrix of 1 with 0 in diagonal \\
\hline$\frac{x_{i}(t)}{M A X_{i}}$ & Degree of adverse impact & Time-dependent \\
\hline $\begin{array}{l}R E_{i}(x) \\
\quad \text { Usually } R E_{i}\left(\frac{x}{M}\right.\end{array}$ & Recovery coefficient of city $i$ & Figure 5 \\
\hline$P O_{i}(t)$ & $\begin{array}{l}\text { Difference between the current and } \\
\text { maximum traffic volumes of each city }\end{array}$ & Time-dependent \\
\hline$r$ & Adjustment parameter in $R_{i}(t)$ & 0.4 \\
\hline$M A X_{i}$ & $\begin{array}{l}\text { Average traffic volume of each city under } \\
\text { normal conditions }\end{array}$ & $\begin{array}{l}\text { Represented by the HPT in the second quarter of } \\
2012 \text { of each city }\end{array}$ \\
\hline
\end{tabular}

Fig. 6 Comparison between observed traffic volume and simulation results in the Beijing-Tianjin-Hebei region, 6 July-5 August, 2012. Relative error is indicated by percentage numbers

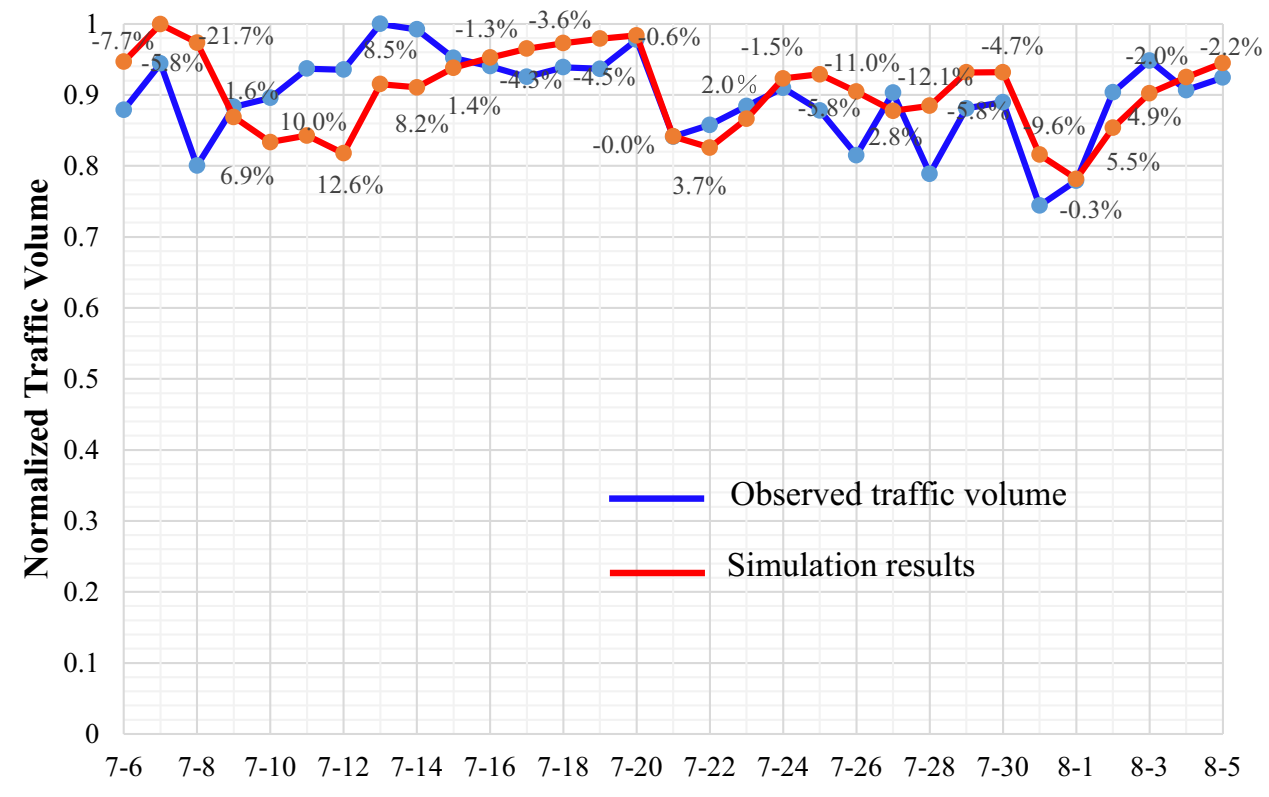

Time
In sensitivity analyses, the trends of the simulation results show a consistent pattern. However, the values of daily traffic volume vary significantly. Several pairs of parameters generate similar results (for example, sets 2 and 3 ; sets 5 and 6; and sets 8 and 9), overlapping each other in Fig. 7. The similarity indicates that the influence of 
Fig. 7 Results of the parametric analyses for $r$ (adjustment parameter) and $B$ (breakdown threshold) with comparison to observed traffic volume in the Beijing-TianjinHebei region, 6 July-5 August, 2012

Fig. 8 Variance of the daily traffic volume simulation results for different parameter settings in the Beijing-Tianjin-Hebei region, 6 July-5 August, 2012
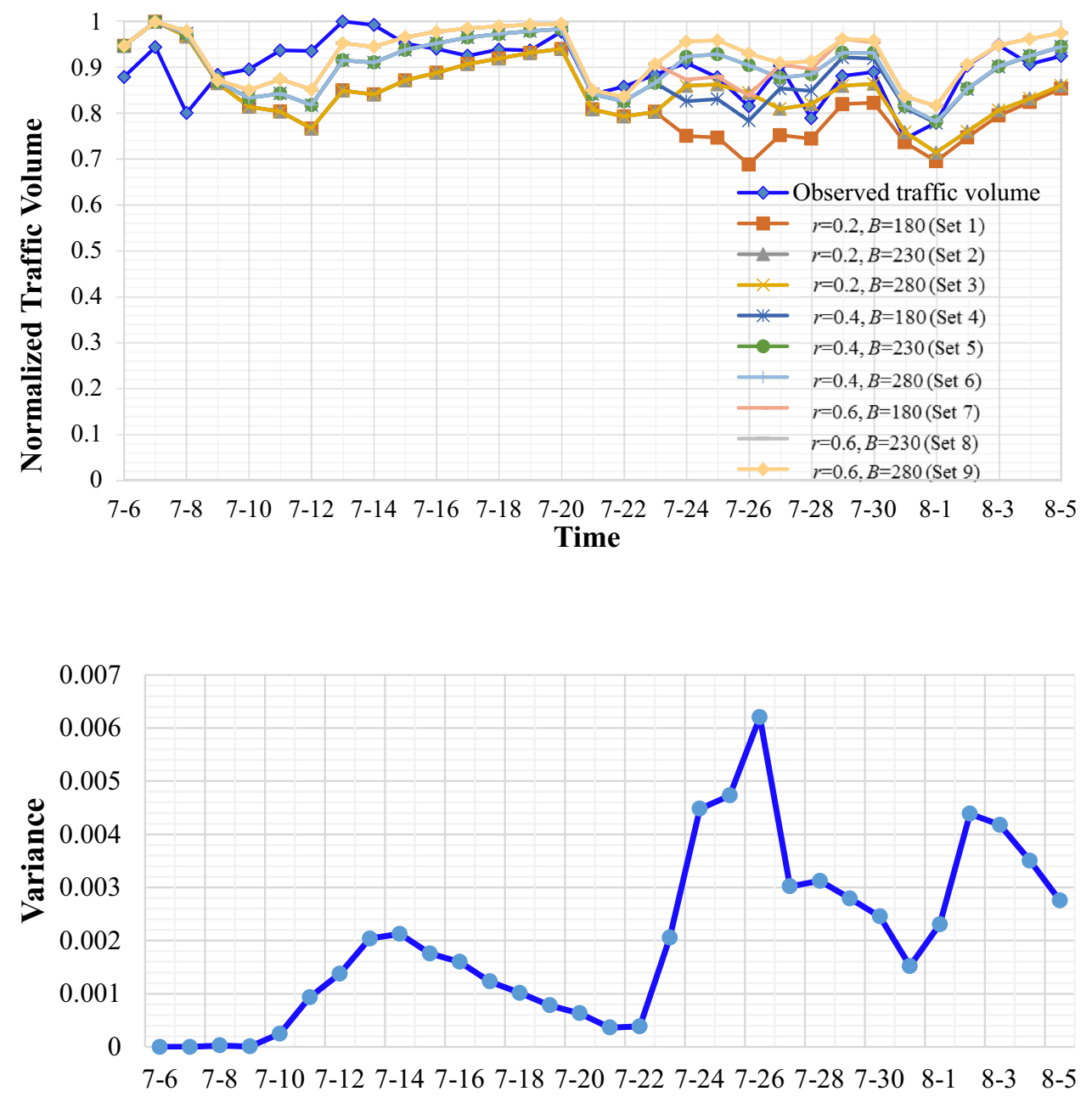

Time variable $B$ is not very significant. This is because in the simulation process of AWORS, there were very rare cases where the accumulated water surpassed $B$, even when it as set at a low value. The difference of simulated traffic volumes between sets 1 and 6 is about $30 \%$ during the days between 24 July and 30 July, which means the influence of $r$ is more significant, especially for days with severe rainstorms. When $r$ is too small the model will overestimate the adverse impact of rainstorms on traffic volume, and when $r$ is too large the model will underestimate the adverse impact.

For each time series, the maximum error rate (compared to observed traffic volume) was $22.4 \%$, while the average error rate was $6.9 \%$. In $6.3 \%$ of the days relative error exceeded $20 \%$, while in $15 \%$ of the days relative error was between 10 and $20 \%$. Most simulation days (73\%) had a relative error of less than $10 \%$. Thus, the parametric analyses confirm the robustness of the proposed model.

In Fig. 8, the variance of the simulated daily traffic volumes under different parameter settings was calculated.
The simulation results begin to diverge around the 5th data point (10 July) and converge again around the 17th data point (22 July). This divergent-convergent behavior repeats over the study period. Such change may be a reflection of the variation in the precipitation for different cities in the case study area.

As shown in Fig. 9, there were six data points with significant variation of daily precipitation between different cities: the 5th (10 July), 17th (22 July), 21st (26 July), 25th (30 July), 27th (1 Aug) and 30th (4 Aug) data points. The simulation results for different parameter settings begin to diverge on the 5th and converge on the 17th data points. When the variance in precipitation was significant, the variance in the simulation results also increased. We assumed some fixed conditions for all cities, such as drainage, recovery ability, and resilience. The covariance between the variance of precipitation and the simulation results suggests that it is necessary to consider the intrinsic differences among cities to improve the accuracy of the model. 
Fig. 9 Variance in daily precipitation for different cities in the Beijing-Tianjin-Hebei region, 6 July-5 August, 2012

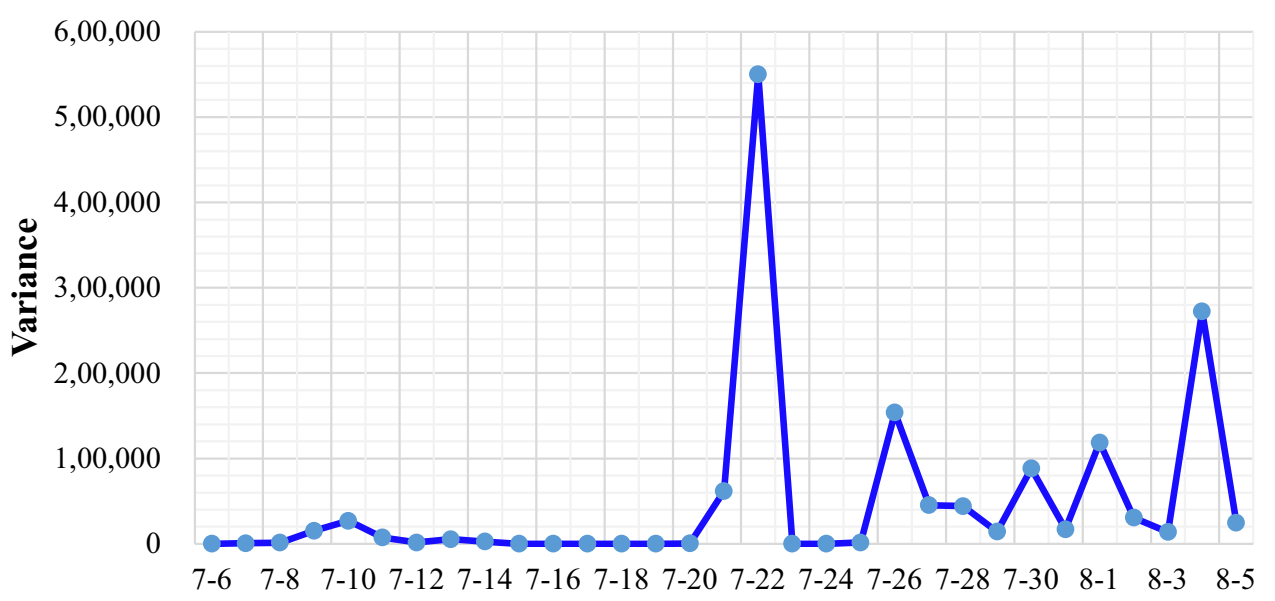

Time
The results indicate that this model has a satisfactory performance and level of abstraction but can still gain reasonable accuracy. A range of parameter values can provide similar model behavior. Different parameter settings bring similar average accuracy but the values for critical days exhibit divergence, which agrees with the findings of Bloomfield et al. (2009). The model efficiently simulates the status of traffic volumes in the case study region. This suggests that the model can be used for rapid assessment of the influence of heavy precipitation over a limited period. It also facilitates rapid assessment of economic losses and recovery planning after such disasters. Furthermore, using predictive data for meteorological hazards, the model can be used as a basis for regional disaster risk analyses and policy making for disaster risk reduction. The structure of the proposed model allows it to be extended to other meteorological hazards or exposures.

\section{Conclusion}

Rapid assessment of the adverse impact of rainstorms on city transport networks is a typical interdisciplinary topic, which requires holistic understanding of the interactions between the networks and meteorological hazards, as well as any intrinsic system properties of a network. In this study, we combined the knowledge from the fields of disaster risk research, transportation engineering, and network systems and developed a mesoscopic model to assess the adverse impact of rainstorms through quantitatively estimating traffic volume in a regional transport network. The proposed impact assessment model takes into account the direct and secondary impacts of rainstorms on transport infrastructures, as well as the indirect effect caused by network connectivity. We used the Beijing-Tianjin-Hebei region as the case study area and simulated the impact of the 21 July 2012 storm event. We evaluated the impact of rainstorms on the regional transport network using traffic data obtained from toll booths. The results show reasonable accuracy and computational efficiency, which demonstrate the potential of the proposed model for rapid disaster impact assessment and risk reduction planning.

The scope of this model can be improved by using more detailed information for individual cities as well as other empirical studies. Aside from the precipitation data, all other parameters in the proposed model were preset and static. We did not consider other dynamic changes in network properties. For example, drainage in cities can be significantly improved with investment in critical infrastructures. In addition, the impedance between cities and the attractiveness of their destination nodes in the gravity model may vary with time. The selection of functions and calibration of parameters were carried out using empirical studies for other areas, and we could improve the accuracy of our results using data from the case study region as they become available. Moreover, we only considered the influence of precipitation, ignoring potential and compounding effects of concurrent meteorological hazards, which could be explored in future research.

Acknowledgments This study was sponsored by the National Science Foundation of China Youth Project (\#41401599), the National Basic Research Program of China (2012CB955402), the Beijing Municipal Science and Technology Commission (Z151100002115040), the International Cooperation Project (2012DFG20710), and the International Center of Collaborative Research on Disaster Risk Reduction.

Open Access This article is distributed under the terms of the Creative Commons Attribution 4.0 International License (http://crea tivecommons.org/licenses/by/4.0/), which permits unrestricted use, distribution, and reproduction in any medium, provided you give appropriate credit to the original author(s) and the source, provide a link to the Creative Commons license, and indicate if changes were made. 


\section{References}

Amin, M. 2000. Toward self-healing infrastructure systems. Computer 33(8): 44-53.

Attoh-Okine, N.O., A.T. Cooper, and S. Mensah. 2009. Formulation of resilience index of urban infrastructure using belief functions. Systems Journal 3(2): 147-153.

Baidu Map. n.d. http://map.baidu.com. Accessed 6 Mar 2015.

Bloomfield, R., L. Buzna, P. Popov, K. Salako, and D. Wright. 2009. Stochastic modelling of the effects of interdependencies between critical infrastructures. Proceedings of the 4th international workshop, critical information infrastructures security, 30 September-2 October 2009, Bonn, 201-212.

Buzna, L., K. Peters, and D. Helbing. 2006. Modelling the dynamics of disaster spreading in networks. Physica A: Statistical Mechanics and its Applications 363(1):132-140.

Camacho, F.J., A. García, and E. Belda. 2010. Analysis of impact of adverse weather on freeway free-flow speed in Spain. Transportation Research Record: Journal of the Transportation Research Board 2169(1): 150-159.

Chang, S.E., T.L. McDaniels, J. Mikawoz, and K. Peterson. 2007. Infrastructure failure interdependencies in extreme events: Power outage consequences in the 1998 ice storm. Natural Hazards 41(2): 337-358.

Chang, S.E., T. McDaniels, J. Fox, R. Dhariwal, and H. Longstaff. 2014. Toward disaster-resilient cities: Characterizing resilience of infrastructure systems with expert judgments. Risk Analysis 34(3): 416-434.

China News. n.d. News communication: 21 July storm caused economic losses of 11.64 billion yuan in Beijing. http://www. chinanews.com/gn/2012/07-25/4058908.shtml. Accessed 6 Mar 2015 (in Chinese).

Chung, Y. 2012. Assessment of non-recurrent congestion caused by precipitation using archived weather and traffic flow data. Transport Policy 19(1): 167-173.

Chung, E., O. Ohtani, H. Warita, M. Kuwahara, and H. Morita. 2006. Does weather affect highway capacity. In Proceedings of the symposium on highway capacity, Yokohama. http://its.iis.utokyo.ac.jp/pdf/5th\%20ISHC\%20Yokohama\%202006\% 20Chung.pdf. Accessed 6 Mar 2015.

Dehman, A. 2012. Effect of inclement weather on two capacity flows at recurring freeway bottlenecks. Transportation Research Record: Journal of the Transportation Research Board 2286(1): 84-93.

Godschalk, D.R. 2003. Urban hazard mitigation: Creating resilient cities. Natural Hazards Review 4(3): 136-143.

Guo, C., H. Xiao, H. Yang, and Q. Tang. 2015. Observation and modeling analyses of the macro-and microphysical characteristics of a heavy rain storm in Beijing. Atmospheric Research 156: $125-141$

Henry, D., and J.E. Ramirez-Marquez. 2012. Generic metrics and quantitative approaches for system resilience as a function of time. Reliability Engineering \& System Safety 99: 114-122.
Hoel, L.A., N.J. Garber, and A.W. Sadek. 2007. Transportation infrastructure engineering: A multimodal integration. Chicago: Thomson Nelson.

Jiang, X., H. Yuan, M. Xue, X. Chen, and X. Tan. 2014. Analysis of a heavy rainfall event over Beijing during 21-22 July 2012 based on high resolution model analyses and forecasts. Journal of Meteorological Research 28: 199-212.

Johansson, J., and H. Hassel. 2010. An approach for modelling interdependent infrastructures in the context of vulnerability analysis. Reliability Engineering \& System Safety 95(12): $1335-1344$.

Munson, B.R., D. Young, and T.H. Okiishi. 2006. Fundamentals of fluid mechanics, 5th edn. New York: Wiley.

National Bureau of Statistics of China. n.d. National data. http://data. stats.gov.cn/. Accessed 6 Mar 2015 (in Chinese).

Rahmstorf, S., and D. Coumou. 2011. Increase of extreme events in a warming world. Proceedings of the National Academy of Sciences of the United States of America 108(44): 17905-17909.

Rinaldi, S.M., J.P. Peerenboom, and T.K. Kelly. 2001. Identifying, understanding, and analyzing critical infrastructure interdependencies. Control Systems 21(6): 11-25.

Saberi, M., and R.L. Bertini. 2010. Empirical analysis of the effects of rain on measured freeway traffic parameters. 89th Annual Meeting of the Transportation Research Board, Washington, DC. http://trid.trb.org/view.aspx?id=910448. Accessed 6 Mar 2015.

Santos, J.R., L.C. Herrera, K.D.S. Yu, S.A.T. Pagsuyoin, and R.R. Tan. 2014. State of the art in risk analysis of workforce criticality influencing disaster preparedness for interdependent systems. Risk Analysis 34(6): 1056-1068.

Smith, B.L., K.G. Byrne, R.B. Copperman, S.M. Hennessy, and N.J. Goodall. 2004. An investigation into the impact of rainfall on freeway traffic flow. 83rd Annual Meeting of the Transportation Research Board, Washington, DC. http://people.virginia.edu/ $\sim$ njg2q/TRB_2004.pdf. Accessed 6 Mar 2015.

Su, B., H. Huang, and Y. Li. 2016. Integrated simulation method for waterlogging and traffic congestion under urban rainstorms. Natural Hazards 81(1): 23-40.

Weng, J., L. Liu, and J. Rong. 2013. Impacts of snowy weather conditions on expressway traffic flow characteristics. Discrete dynamics in nature and society 2013. Article 791743.

World Meteorological Organization (WMO). 2011. WMO statement on the status of the global climate in 2010. WMO-No. 1074. http://www.wmo.int/pages/prog/wcp/wcdmp/statement/documents/ 1074_en.pdf. Accessed 6 Mar 2015.

Yang, S.N., J.Y. Ye, X.C. Zhang, and H. Liu. 2012. Study of the impact of rainfall on freeway traffic flow in Southeast China. International Journal of Critical Infrastructures 8(2/3): $230-241$ 